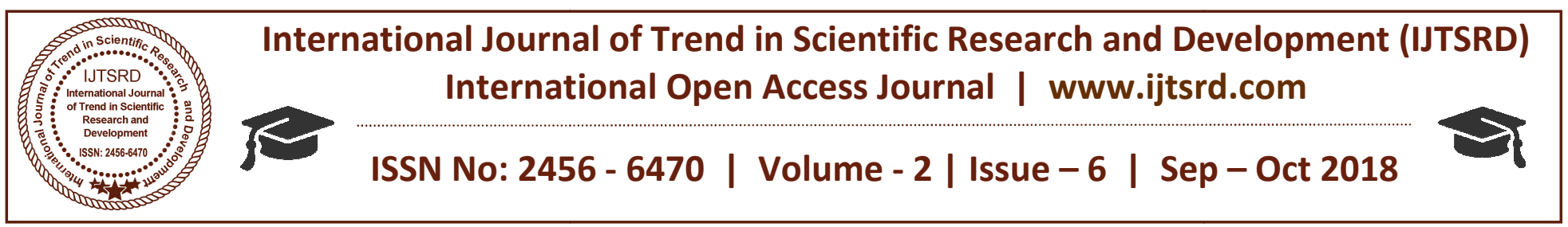

\title{
Multimodal Biometrics Authentication System using Fusion of Fingerprint and Iris
}

\author{
Miss. Kamble Sunayana Nivrutti, Prof. Gund. V. D., Prof. Kazi K. S \\ Department of Electronics and Telecommunication Engineering, \\ Brahmdevdada Mane Institute of Technology, Solapur, Maharashtra, India
}

\begin{abstract}
In the present era of information technology, there is a need to implement authentication and authorization techniques for security of resources. There are number of ways to prove authentication and authorization. But the biometric authentication beat all other techniques. Biometric techniques prove the authenticity or authorization of a human being based on his/her physiological or behavioural traits. It also protects resources access from unauthorized users. We will develop a multimodal biometric identification system that represents a valid alternative to conventional approaches. In biometric system physical or behavioural traits are used. A multimodal biometric identification system aims to fuse two or more physical or behavioural traits. Multimodal biometric system is used in order to improve the accuracy. Multimodal biometric identification system based on Iris \& fingerprint trait based on fuzzy logic is proposed. Typically in a multimodal biometric system each biometric trait processes its information independently. The processed information is combined using an appropriate fusion scheme.
\end{abstract}

Keyword: Iris, Finger print, collaret, Hough, Haar.

\section{INTRODUCTION}

The term biometrics is derived from the Greek words Bio \& Metric. The term Biometrics relates to the measurement (metric) of characteristics of a living (Bio) thing in order to identify a person. Biometrics uses various physiological or behavioural characteristics. Common physiological biometric measurements include fingerprints, hand geometry, retina, iris, facial images etc. While common behavioural biometric measurements include signatures, voice recordings, keystroke rhythms etc. [1]
Biometric characteristics can be divided in two main classes:

$>$ Physiological are related to the shape of the body. Examples include, but are not limited to fingerprint, face recognition, DNA, Palm print, hand geometry, iris recognition, which has largely replaced retina, and odour/scent.

Behavioural are related to the behaviour of a person. Examples

Include, but are not limited to typing rhythm, gait, and voice \& handwritten signatures. Some researchers have coined the term behaviour-metrics for this class of biometrics [1]

\section{IRIS RECOGNITION}

Iris is unique to each individual and remains constant over the life of a person. The eyeball has a circular black disk in the centre known as pupil. The pupil dilates when exposed to light and contracts in dark. Thus the size of pupil varies with respect to light it is exposed to. The iris is the annular ring between the sclera and pupil boundary and contains the flowery pattern unique to each individual. This texture information unique to each individual is extracted from rest of the eye image and is transformed into strip to apply pattern matching algorithm between the database and query images of iris.

In this work a combination of two classification methods are proposed rather than a single method. The zigzag collaret area of the iris is selected for iris feature extraction because it captures the most important areas of iris complex pattern. [6] A parabola detection technique is used for eyelid detection and a median filter is used for eyelash removal. HAAR wavelet of decomposition level 3 and 
1D Log Gabor filter is used for feature extraction. For the purpose of classification support vector machine is used as a main classifier while Hamming distance is used as a secondary classifier. By combining these two classification techniques (SVM and Hamming distance) we achieved an accuracy of $99.91 \%$ and 99.88\% on CASIA (CASIA iris image database ) and Check iris image database (Check Image Database). The proposed authentication system is divided in two phase. In the first phase, iris patterns need to record, is referred as an enrolment phase. At the time of identification use stored iris image features, this is called identification phase.

The second is to find the zigzag collaret area of the iris. The last step is to locate the eyelids in the eye image.

\section{HOUGH TRANSFORM}

The Hough transform is a technique that can be used to determine the parameters of simple geometric objects, such as lines and circles present in an image.

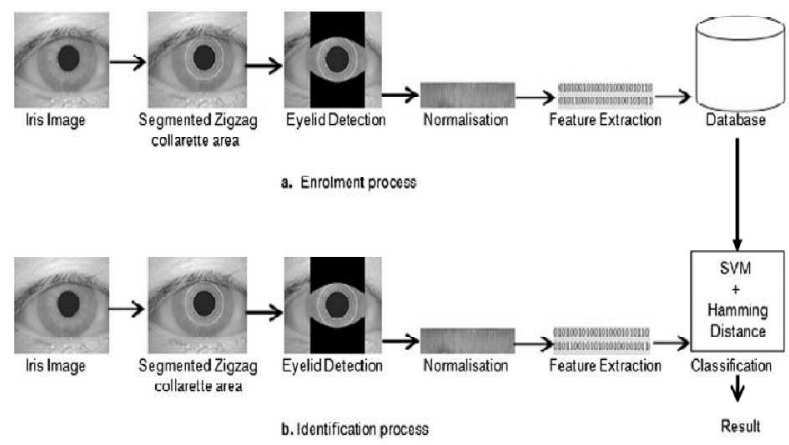

Fig.1 Flow diagram of proposed system

The circular Hough transform can be employed to deduce the centre and radius coordinates of the pupil and iris regions (Masek, 2003) when a number of points that fall on the perimeter are known. A circle with radius $r$ and centre $(x c, y c)$ can be described by the parametric equations [3]

$$
\begin{aligned}
& x=x_{c}+r * \cos \theta \\
& y=y_{c}+r * \sin (\theta)
\end{aligned}
$$

When the angle $\mathrm{h}$ sweeps through the full $360^{\wedge}$ Orange, the points $(x, y)$ trace the perimeter of a circle. If an image contains many points, some of which fall on the perimeters of circles, then the job of the search program is to find co-ordinate triplets $(\mathrm{xc}, \mathrm{yc}, \mathrm{r})$ to describe each circle. In order to simplify the parametric representation of the circle, the radius can be held as a constant or limited to a number of known radii. The process of finding circles in an image using circular Hough transform is: First we find all edges in the image. This step has nothing to do with Hough Transform and any edge detection technique can be used.

For all edge points (xi, yi), i = 1,2,. . .,n. A Hough transform can be written as

$$
\begin{aligned}
& H\left(x_{c}, y_{c}, r\right)=\sum_{i=1}^{m} h\left(x_{i}, y_{i}, x_{c}, y_{c}, r\right) \\
& h\left(x_{i}, y_{i}, x_{c}, y_{c}, r\right)=\left\{\begin{array}{ll}
1, & \text { if } g\left(x_{i}, y_{i}, x_{c}, y_{c}, r\right)=0 \\
0, & \text { otherwise }
\end{array}\right\}
\end{aligned}
$$

where $g\left(x_{i}, y_{i}, x_{c}, y_{6}, r\right)=\left(x_{i}^{2}-x_{c}^{2}\right)+\left(y_{i}^{2}-y_{6}^{2}\right)-r^{2}$. The three co-ordinates $\left(x_{0} y_{0} r\right)$ for which $H\left(x_{0} y_{c} r\right)$ is highest will become the coordinate of centre and radius of the circle.

\section{ISOLATION OF ZIGZAG COLLARET AREA}

The zigzag collaret area of the iris was selected for iris feature extraction because it captures the most important areas of iris complex pattern. It is not very much affected by eyelids and eyelashes because it was closed with the pupil. The previously obtained centre value of pupil was used for detection of collaret zigzag area because it is generally concentric to pupils and radius of this part of the iris was restricted in a certain range. The detected collaret zigzag area on the Check image database is shown in Fig. 2

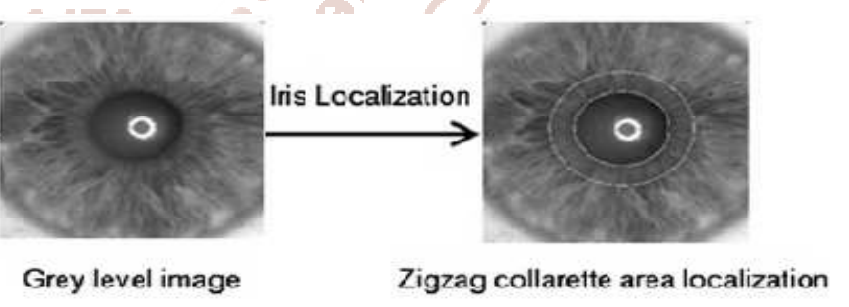

Fig.2 Zigzag collaret area localisation on the Check image database.

Two search regions were selected for the purpose of upper and lower eyelids detection. The search regions are confined within the pupil and zigzag collaret area of the iris.

\section{Width of search region =radius of iris (zigzag area) - radius of pupil}

A horizontal edge map was used to find an edge image of an eye image as eye lid part is present in upper or lower horizontal region. At each edge point within the search regions, a parabolic Hough transformation was applied for eyelids detection. 


\section{NORMALISATION AND EYELASH REMOVAL}

A method for removing eyelashes and restoring the underlying iris pattern as much as possible is developed. In this step the zigzag collarette area pixels which occluded by eyelashes were recreated using information from their non-occluded neighbours. For every pixel $(x, y)$ present in the normalised image we first decide whether the pixel is occluded by eyelash or not.[5]

\section{If $I(x ; y)<T ;$ pixel is occluded by eyelash}

Where $\mathrm{I}$ is the intensity of a pixel and $\mathrm{T}$ is a threshold. For every pixel which satisfies above equation, a 5 x 5 median filter is applied on that pixel. In $5 \times 5$ neighbourhoods only those pixels which are greater than $\mathrm{T}$ are selected because they are not occluded by an eyelash. At last we sort all pixels in ascending order and centre pixel value was replaced with the median value of $5 \times 5$ window neighbourhoods [5]

\section{FEATURE EXTRACTION}

For the purpose of classification; support vector machine was used as a main classifier while Hamming distance was used as a secondary classifier.

\section{HAAR WAVELET DECOMPOSITION}

In our work HAAR wavelet is applied to the normalised image of size $64 \times 512$ at three different levels successively for feature extraction. The wavelet transform has been performed and the image is divided into four sub-regions LL, HL, LH, and HH. Most of the energy is contained in LL (low frequency) region on the multi-divided iris image, since the extracted region has contained major information on the iris image; this LL region (sub-image) is provided as an image to be newly processed so that we can again apply the wavelet transform to the relevant region. The HAAR wavelet transform is repeatedly performed in order to reduce information sizes as shown in Fig.4. In this way, the characteristic values of further reduced region such as LL3 are obtained (Patil \& Patilkulkarani, 2009). In this work, the region LL3 for each iris image is obtained by performing the wavelet transforms three times which is considered as a major characteristic region. The values of LL3 region are used as components of the characteristic input vector to SVM. At this time, the region LL3 contains the information having $8 \times 64=512$ features. [4]

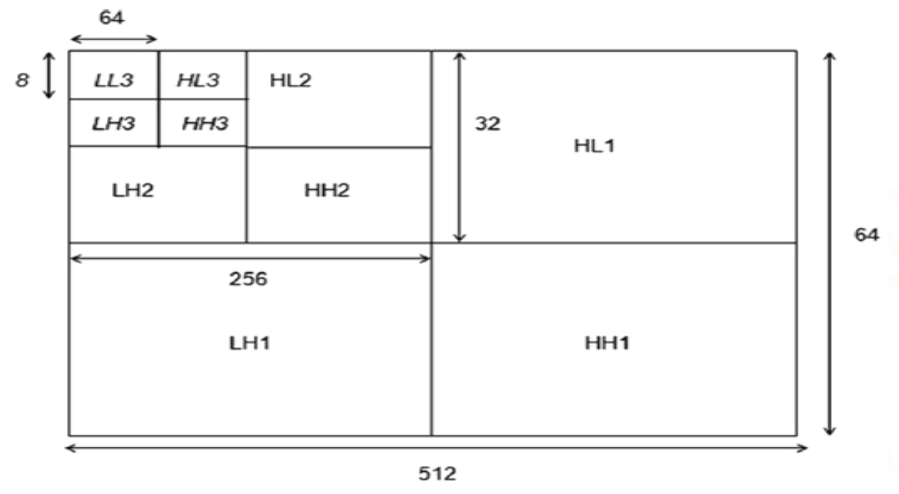

Fig.4 HAAR three level wavelet decomposition.

\section{Fingerprint minutiae Extraction}

Image Enhancement:-Histogram Equalization and Fourier Transformer adopted in fingerprint enhancement. Histogram equalization is to expand the pixel value distribution of an image so as to increase the perception information, and Fourier transform divide the image into small processing blocks (32by 32 pixels) and In order to enhance a specific block by its dominant frequencies, multiply the FFT of the block by its magnitude a set of times and get Enhanced fingerprint image

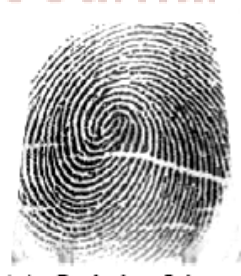

(a) Original image

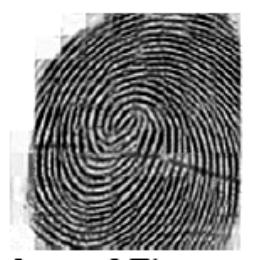

(b) Enhanced Fingerprint

\section{Fig.3 Enhanced Fingerprint image}

Enhanced fingerprint image is than binarized as In Fingerprint Image binarization is process to transform gray image into binary image in system it use for transform the 8-bit Gray fingerprint image to a 1-bit image with 0 -value for ridges and 1-value for furrows. After the operation, ridges in the fingerprint are highlighted with black colour while furrows are white. A locally adaptive binarization method is performed to binaries the fingerprint image. Such a named method comes from the mechanism of transforming a pixel value to 1 if the value is larger than the mean intensity value of the current block is $16 \times 16$ to which the pixel, then from the binarized image estimates the direction for fingerprint.

\section{MINUTIAE EXTRACTION}

For minutia extraction stage, three thinning algorithms are tested and the Morphological thinning operation is finally given out with high efficiency and pretty good thinning quality. For this stage, a more 
rigorous algorithm is developed to remove false minutia based. Also a new co-ordinate representation for bifurcations in fingerprint is proposed to unify terminations and bifurcations.[2]

\section{RIDGE THINNING}

Ridge Thinning is to removes the redundant pixels of ridges till the ridges are just one pixel narrow. In each scan of the full fingerprint image, the algorithm marks down redundant pixels ineach small image window (3x3 pixel). And finally removes all those marked pixels after several scans.[2]

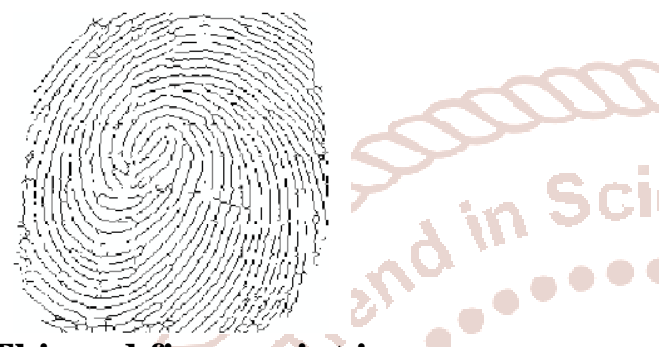

Fig.5 Thinned fingerprint image

Thinned fingerprint image: - After the fingerprint ridge thinning, marking minutia points is relatively easy. But it is still not a trivial task as most literatures declared because at least one special case evokes my caution during the minutia marking stage. Minutia marking stage where mark the ridged and bifurcation of fingerprint. Minutiae are extracted by scanning the $3 \times 3$ window across each ridge pixel which determines the $\mathrm{CN}$ value is use in many system in this it consider 8 neighbours, but in this system divide thinned image in $16 \times 16$ processing block and perform the scanning and it have a $3 \times 3$ window. Then interest in find the Neighbours' Value. Neighbour value (NV) is calculated and then finds the number of Neighbours' $(\mathrm{NN})$ as equations:

$$
\begin{aligned}
& N V=\sum_{i=-1}^{1} \sum_{j=-1}^{1}\left(p_{i}, p_{j}\right) \\
& N V=N V-1
\end{aligned}
$$

This number of neighbour's value is determines a ridge pixel either to be an ending, bifurcation or nonminutiae point. The minutia marking, all thinned ridges in the fingerprint image are labelled with a unique ID for further operation. [4]

\section{FUSION}

Fusion is performed by combining two feature vectors extracted from every pair of fingerprints and irises and stored in the mat file using append operation of mat lab. The homogenous biometric vector from fingerprint and iris data is composed of binary sequences representing the unimodal biometric templates. The proposed approach is based on pair recognition of fingerprint and iris, and every part provides its own Matching score. Then Iris and Fingerprint Matching score is fused using simple sum rule. If the Fused matching score is larger than a prespecified threshold, then the parson is accepted or rejected so it is clear that nobody can be accepted unless fused score of the results are greater than prespecified threshold. Matching score for Iris is calculated through Hamming distance (HD) between two final fused templates.[4]

$$
\mathrm{HD}=\frac{1}{\mathrm{~N}} \sum_{\mathrm{i}=1}^{\mathrm{N}} \mathrm{XOR}\left(\mathrm{X}_{\mathrm{A}}(\mathrm{i}), \mathrm{Y}_{\mathrm{B}}(\mathrm{i})\right)
$$

Where, and are the coefficients of two iris images and $\mathrm{N}$ the size of the feature vector. For fingerprint minutia matcher it has two stages Alignment stage and Match stage. The matching algorithm for the aligned minutia patterns needs to be elastic since the strict match requiring that all parameters $(\mathrm{x}, \mathrm{y}, \theta)$ are the same for two identical minutias is impossible due to the slight deformations and in exact quantization of minutia.

$$
M S=F_{\text {iris }}+F_{\text {Fingerprint }}>\text { Threshold }
$$

The elastic matching of minutia is achieved by placing a bounding box around each template minutia. If the minutia to be matched is within the rectangle box and the direction discrepancy between them is very small, then the two minutias are regarded as a matched minutia pair. Each minutia in the template image either has no matched minutia or has only one corresponding minutia. The final match ratio for two fingerprints is the number of total matched pair over the number of minutia of the template fingerprint. The score is $100 *$ ratio. Then matching score of fingerprint is fused with matching score of iris using sum rule.

\section{RESULT ANALYSIS:}

\begin{tabular}{|c|c|c|}
\hline & (existing) & (Proposed) \\
\hline efficiency & 50 & 65 \\
\hline availability & 60.5 & 70.2 \\
\hline performance & 70.56 & 85.78 \\
\hline Accuracy & 93.4 & 95.9 \\
\hline scalability & 90.5 & 90.5 \\
\hline
\end{tabular}




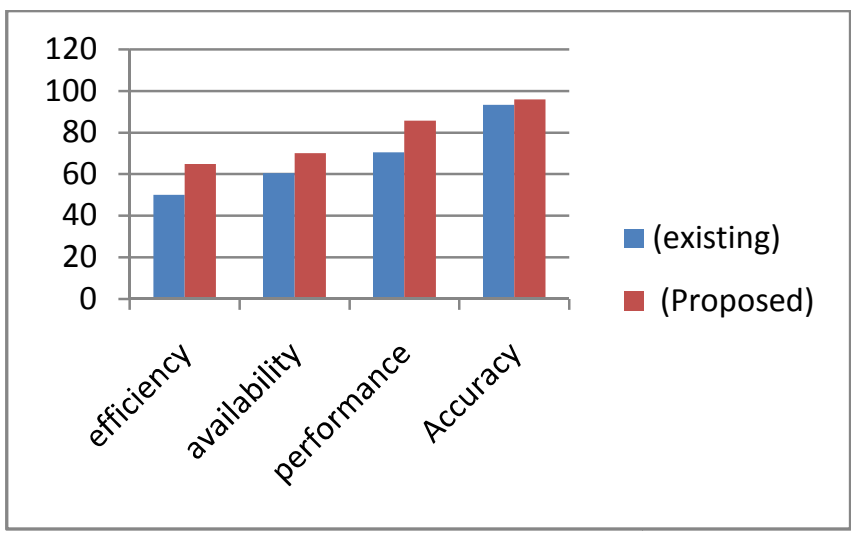

\section{CONCLUSION}

The basic approach of this system is to design a secure and effective technique for personal authentication on noisy iris \& fingerprint recognition. For experimentation and implementation a dataset of a database has been used and evaluation results clearly demonstrate that the proposed iris \& fingerprint segmentation technique provides better accuracy in iris \& fingerprint recognition system performance with capturing in non-ideal conditions. In proposed system, we will authenticate the person with the available database by fusing sclera features with iris for relaxed image capturing could lead to better recognition rates with low cost. The texture features are extracted by using the SVM algorithm and then extracted feature vectors are classified by using SVM algorithm. SVM offers a computationally easier model to get the solution which is global minimum and unique. The research results indicate that fusion of iris and finger prints are better than their individual performances. The proposed method can work effectively and objectively.

\section{REFERENCES}

1. Dr. Vinayak Ashok Bharadi Bhavesh Pandya, Mr. Bhushan Nemade CONFERENCE PAPER. SEPTEMBER 2014 https://www.researchgate.net/publication/2674352 42

2. Dipti. S. Randive Manasi. M. patil, Ph.D International Journal of Computer Applications (0975 - 8887) Volume 77 - No.11, September 2013

3. Himanshu Rai, Anamika Yadav Expert Systems with Applications (2013) journal homepage: www.elsevier.com/locate/eswa

4. Hasimah Ali Momoh Salami Iris Recognition System Using Support Vector Machines Chapter . October2011 https://www.researchgate.net/publication/2219184 32

5. M. Rizky Faundra1 and Dwi Ratna Sulistyaningrum2 Iris Segmentation and Normalization Algorithm Based on Zigzag Collarette IOP Conf. Series: Journal of Physics: $\begin{array}{llll}\text { Conf. Series } \quad 795 & \text { (2017) } 012049\end{array}$ doi:10.1088/1742-6596/795/1/012049

6. Roy, K., \& Bhattacharya, P. (2006). An iris recognition method based on zigzag collarette area and asymmetrical support vector machines. IEEE International Conference on Systems, Man and Cybernetics, 1, 861-865. 